\title{
The Effect of the PRF and Simvastatin on Bone Formation and Inflammatory Markers after Induction of Critical Size Bone Defects
}

ISSN: 2576-8875

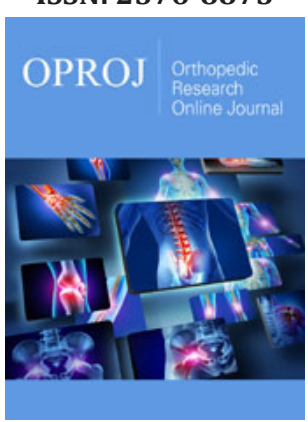

*Corresponding author: Shereen Raafat N, Department of Pharmacology and Toxicology, The British University in Egypt (BUE), Egypt

Submission: 僵 December 12, 2019

Published: 䟧January 06, 2020

Volume 6 - Issue 3

How to cite this article: Shereen Raafat N, Reham Amin M, Elmazarc M, Mahmoud Khattab M, Aiman El-Khatib S Factors Affecting Parents to 'Drop-Out' from Ponseti Method and Children's Clubfoot Relapse. Ortho Res Online J. 6(3) OPROJ.000637.2020.

DOI: 10.31031/OPROJ.2020.06.000637

Copyright@: Shereen Raafat N, This article is distributed under the terms of the Creative Commons Attribution 4.0 International License, which permits unrestricted use and redistribution provided that the original author and source are credited.
Shereen Raafat $\mathbf{N}^{1 *}$, Reham Amin $\mathbf{M}^{2}$, Elmazarc $\mathbf{M M}^{` 3}$, Mahmoud Khattab $\mathbf{M}^{4}$ and Aiman El-Khatib $S^{4}$

${ }^{1}$ Department of Pharmacology and Toxicology, Faculty of Dentistry, The British University in Egypt (BUE), Egypt

${ }^{2}$ Department of Oral Biology, Faculty of Dentistry, The British University in Egypt (BUE), Egypt

${ }^{3}$ Department of Pharmacology and Toxicology, Faculty of Pharmacy, The British University in Egypt (BUE), Egypt.

${ }^{4}$ Department of Pharmacology and Toxicology, Faculty of Pharmacy, Cairo University, Egypt

\section{Abstract}

Aim: This study compares the bone regenerative power of SIM and PRF and the combination added locally on induced bone defect and their effect on inflammatory markers.

Material and method: A critical size bone defect was induced in 48 male albino rats of average weight 150-200g and were divided into 4 groups according to the filling material. Control, PRF, SIM, and SIM/ PRF group. Each group was subdivided according to the sacrificing period into two subgroups (one and two-months postoperatively). Tibial specimens were evaluated histologically using Hematoxylin and eosin (H\&E) stain, serum inflammatory markers (IL-1 $\beta$, IL-6, IL-10 and TNF- $\alpha$ ) 6 days post-surgery using ELISA technique.

Result: Bone specimens of the SIM group and the combination showed well observed darkly stained areas of matured bone compared to the other groups especially two-month postoperatively, the SIM/PRF group showed matured bone trabeculae surrounding bone marrow spaces which appeared densely stained with normal osteocytes lacunae. Resting and reversal lines were obviously detected in the combination group only, denoting high bone remodeling activity in this group. PRF and SIM decreased significantly the pro-inflammatory IL-1 $\beta$ serum levels compared to the control group, SIM loaded on PRF showed the highest statistically significant decrease in IL- $1 \beta$ serum concentration $(\mathrm{P}<0.001)$. IL- 6 and TNF- $\alpha$ serum levels didn't differ significantly between the control and PRF group but showed statistically significant decrease in the SIM and SIM/PRF groups. In contrast PRF, SIM and SIM/PRF significantly increased IL-10 serum levels compared to the control group with the highest significant increase in the SIM/PRF group $(\mathrm{P}<0.001)$.

Conclusion: Comparing SIM with PRF each as a sole filling material in the bone defect, SIM showed more enhanced bone regeneration and more powerful anti-inflammatory effect, while the combination has proven to cause the most significant bone formation but without significant effect on inflammation.

\section{Introduction}

Statins are three-hydroxy-3-methyl-glutaryl-CoA (HMG-CoA) reductase competitive inhibitors, which are commonly used agents for lowering cholesterol and reducing the risk for cardiovascular disease. Recent data suggest that statins influence bone metabolic activity by stimulating new bone formation [1]. Bone resorption in periodontitis, an inflammatory disease, is caused by the interaction of various cytokines in the inflamed tissue, produced by immune cells and also by resident cells as human gingival fibroblasts (HGF) and periodontal ligament (PDL) cells [2]. Three molecules, members of the tumor necrosis factor (TNF) ligand and receptor super families, regulate the process of osteoclast formation. The first one, receptor activator for NF-kB ligand (RANKL) which is expressed on hematopoietic stromal cells and periosteal osteoblasts as well as on HGF and PDL cells. RANKL binds to its corresponding re- 
ceptor, RANK, on mononucleated osteoclast precursors and causes their activation to multinuclear bone resorbing osteoclasts [3]. The effects of RANKL are blocked by its soluble decoy receptor, osteoprotegerin (OPG), thus inhibiting osteoclast activation and differentiation. RANKL and OPG are produced by many cells in the body including PDL and human gingival fibroblasts cells [4].

Studies investigating Atorvastatin have shown that its treatment leads to significant reductions in the levels of pro-inflammatory cytokines (TNF, IL-1 and IL-6) [5]. In another study, Atorvastatin significantly decreased bone resorption markers, including levels of serum IL-6 [6]. Atorvastatin also decreased COX-2 expression within peripheral blood monocytes in patients with acute myocardial infarction [7] and increased IL-10 levels in a dose dependent manner [8]. Atorvastatin has also been used to inhibit metalloproteinases [9], osteoclast genesis and bone destruction, and the expression of the receptor activator of nuclear factor kappa B ligand (RANKL) [10]. Among the great challenges facing clinical research is the development of bioactive surgical additives regulating inflammation and increasing healing. Indeed, after each intervention, surgeons must face complex tissue remodeling phenomena and the consequences on healing and tissue survival [11]. The main goal of the modern surgery is to get a low invasiveness and a high rate of clinical healing. Nowadays, it has been widely introduced the concept of the "regenerative surgery" [12]. Thus, healing is a complex process, which involves cellular organization, chemical signals, and the extracellular matrix for tissue repair. The understanding of healing process is still incomplete, but it is well known that platelets play an important role in both hemostasis and wound healing processes [13].

A few techniques have been utilized in modern dentistry to speed the regeneration of either hard or soft tissues [14]. Guided Bone Regeneration (GBR) is a procedure that enables the regeneration of the bone volume through the protection of the blood clot within the bone compartment under a resorbable or non-resorbable membrane [15]. A wide range of biocompatible, biodegradable and nontoxic synthetic or natural biomaterials are used as careers or scaffolds in tissue regeneration, to provide local mechanical strength and to facilitate the process of attachment, proliferation and differentiation of stem and progenitor cells [16]. The use of platelet concentrates has gained increasing awareness in recent years for regenerative procedures in modern dentistry [17].

Platelet concentrates are defined as autologous or allogeneic platelet derivatives with a platelet concentration higher than baseline and they are widely used in different areas of Regenerative Medicine in order to enhance wound healing processes [18]. Platelets regenerative potential was introduced in the 70's, when it was observed that they contain growth factors that are responsible for increase collagen production, cell mitosis, blood vessels growth, recruitment of other cells that migrate to the site of injury, and cell differentiation induction, among others. One of the latest innovations in oral surgery is the use of platelet concentrates for in vivo tissue engineering applications: Platelet Rich Plasma (PRP) and Platelet Rich Fibrin (PRF) [19].

Platelet Rich Fibrin, a rich source of autogenous cytokines and growth factors, can be considered as a healing biomaterial. It has important properties for cicatrization, such as angiogenesis, immune control, attaching the circulating stem cells, and wound protection by epithelial cover. The properties of PRF are considered to promote both soft-tissue and bone regeneration and are suitable for ridge preservation [20]. Indeed, PRF has a therapeutic capacity and its specific structure makes it an appropriate membrane to improve the healing process, and in the future, hopefully will continuing to show more promising clinical results.

\section{Objective}

The purpose of this study compares the bone regenerative power of SIM and PRF and the combination added locally on induced bone defect and their effect on inflammatory markers.

\section{Material and Method}

\section{Animals}

Forty-eight male Sprague Dawley rats (two-month old) weighting between 180-220 grams were used in the present study. The rats were housed in separate cages, three rats per cage in the animal house of "Pharmacology Research Center" in the Faculty of Pharmacy, the British University in Egypt. All animal experiments complied with the arrive guidelines and had been approved by the local Animal Ethical Committee of Faculty of pharmacy, Cairo University, Egypt. Rats were provided rat chow and tap water ad libitum throughout the experimental period.

\section{Simvastatin (SIM) and chemicals}

Simvastatin and gelatin powder were purchased from Sigma-Aldrich (Sigma, ST. Louis, MO, USA). Other Drugs used in this study included; xylazine (Anased; Akorn, Inc., LF, USA), Ketamine (Ketamax; Trikaa ph., Uttarakhand, India), Betadine (Mundipharma ph., Cairo, Egypt), ceftriaxone (Rocephin; Roche, Basel, Switzerland), and diclofenac sodium (Ketofan; Amoun ph. Comp., Cairo, Egypt). Chemicals used in the current study included; Ethylene diamine tetra-acetic acid (EDTA) (Merck, Darmstadt, Germany), formalin (Labsynth, Diadema, SP, Brazil), and phosphate buffer solution (PBS; Sigma, ST. Louis, MO, USA). ELISA kits used included; serum rat IL-1 $\beta$, IL-6 ELISA kit (RayBiotech Inc., Georgia, USA), rat TNF- $\alpha$ ELISA kit (Sigma Aldrich, St. Louis, USA).

\section{PRF and gelatin granules preparation}

To prepare PRF; around $5 \mathrm{ml}$ of human venous blood was collected into two sterile vacutainer tubes $6 \mathrm{ml}$ capacity without anticoagulant. The vacutainer tubes were centrifuged at 3000 revolutions per minute (rpm) for 10 minutes [1]. The upper straw-colored layer was then removed and the middle fraction containing the fibrin clot was collected (Figure 1). 


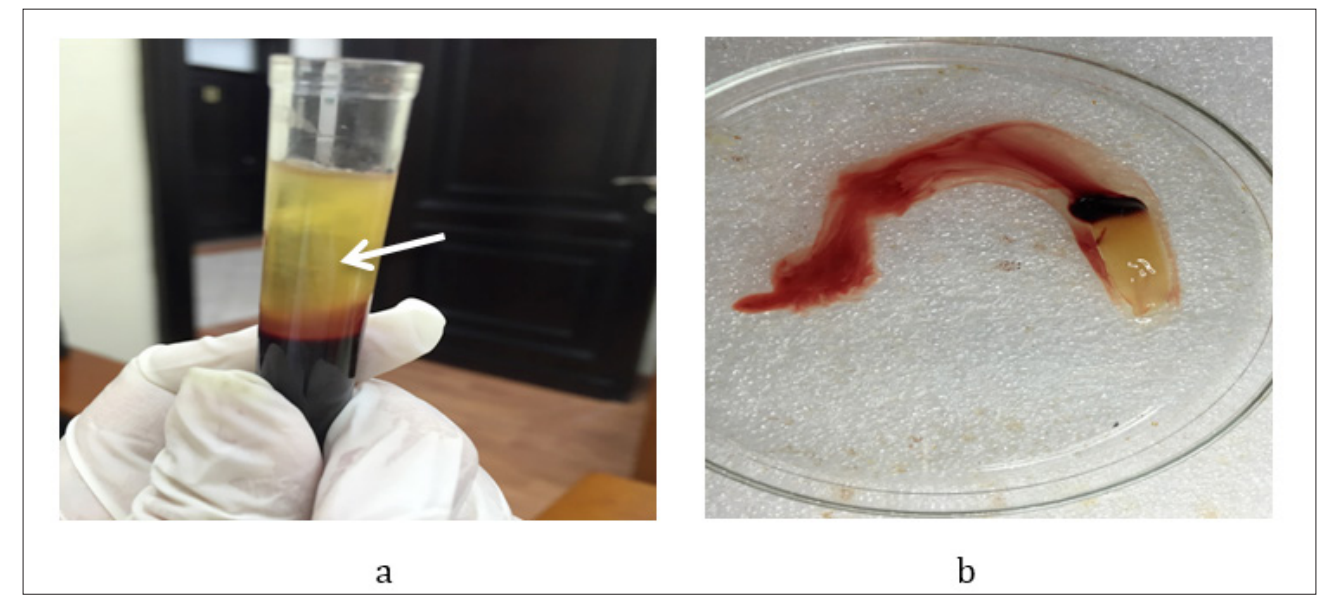

Figure 1: Platelet rich fibrin separation,

a. Shows the three different layers after centrifugation; red lower fraction containing red blood cells, upper straw colored cellular plasma and the middle fraction containing the fibrin clot,

b. Shows PRF clot after separation.

\section{Surgical procedures}

Rats of different groups were anesthetized by intraperitoneal injection of Ketamine hydrochloride $10 \% 33 \mathrm{mg} / \mathrm{Kg}$ body weight) and Xylazine Hydrochloride $2 \%$ (13mg/kg body weight) cocktail. The cocktail was freshly prepared before the surgical operation [18]. Following anesthesia, the surgical site at right tibia was shaved and disinfected with Betadine. Then, a linear incision of $1-1.5 \mathrm{~cm}$ was performed just below the head of the tibia through the skin, fascia and periosteum to expose bone on the lateral surface of the right tibia. Exposure of the bone as well as reflection of periosteum was completed through blunt dissection of the underlying muscle. A monocortical bone defect extended to bone marrow at the middle third of the lateral surface of the right tibia was created under continuous saline irrigation by sterile stainless steel round dental bur size 6 used on a low speed motor (Aseptico; Woodinville, WA, USA). The muscular layer was sutured with resorbable 4.0 catgut (Cirumédica, Cotia, SP, Brazil) and the skin was sutured with interrupted 4.0 silk suture (Ethicon/Johnson \& Johnson, São José dos Campos, SP, Brazil) (Figure 2).

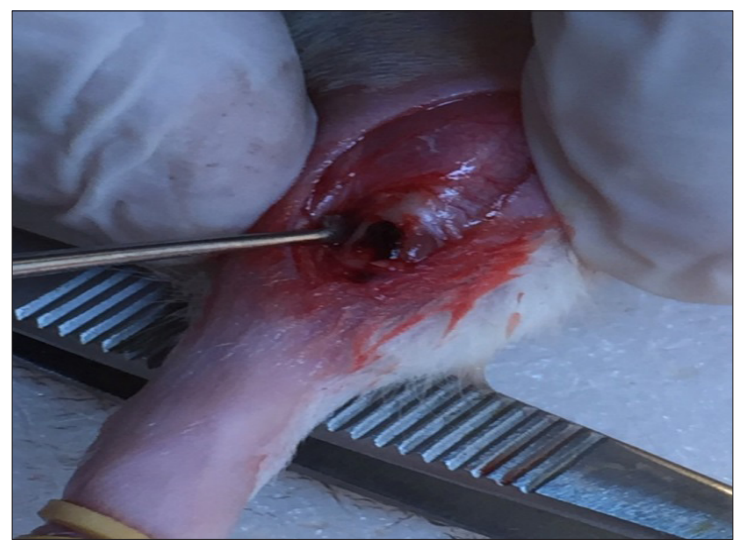

Figure 2: A monocortical bone defect extended to bone marrow at the middle third of the lateral surface of the right tibia.

\section{Experimental design}

The rats were divided randomly into four main groups $(n=12$ per group) according to the material by which the bone defect was loaded. In the control group animals, the bone defect was left empty. In the PRF group, the bone defect was filled with PRF (6mg weight per animal). In the SIM group, the bone defect was filled with $1 \mathrm{mg}$ SIM loaded on gelatin. In the SIM/PRF group the bone defect was filled with $1 \mathrm{mg}$ SIM mixed with PRF (SIM/PRF ratio 1:6 by weight).
At the end of the surgical procedure, each rat received intramuscular injection of ceftriaxone at dose of $20 \mathrm{mg} / \mathrm{kg}$ and diclofenac sodium at dose of $75 \mathrm{mg} / \mathrm{ml}$, once daily for 3 days postoperatively starting immediately after the operation. Animals were sacrificed by an overdose of anesthesia and each group was subdivided into 2 subgroups ( $n=6$ per subgroup) according to sacrificing time; onemonth postoperatively and the remaining animals two-months postoperatively. 


\section{Bone tissue preparation for staining}

\section{Hematoxylin and eosin stain}

\section{A. Bone tissue preparation for staining}

Rats were sacrificed by over dose of anesthesia and then tibiae were dissected free from any soft tissue carefully. The rest of rat's bodies were appropriately gotten rid of them in the incinerator of BUE Dentistry Hospital. Specimens were fixed in 10\% buffered formaldehyde for five days [the formaldehyde was buffered in $\mathrm{PH}$ 7.2 by phosphate buffer saline (PBS). The specimens were decalcified using a solution containing 12\% Ethylene diamine tetra-acetic acid (EDTA) buffered in PH 7.2 by PBS for three weeks at $4{ }^{\circ} \mathrm{C}[21]$.

B. After complete decalcification, specimens were assigned for histological staining

Histological staining was performed to visualize bone formation using Hematoxylin and eosin (H\&E) stain and was done according to the method described by Bancroft et al. [21]. Briefly, after complete decalcification specimens were washed properly under running water, dehydrated by transferring through ascending concentrations of alcohol (50\%, 60\%, 80\%, 90\%, 96\%, and absolute alcohol). Specimens were then infiltrated in paraffin wax blocks. The embedded specimens were sectioned by microtome (3 microns thick), the sections were transferred in descending concentrations of alcohol $(96 \%, 70 \%$, and then distilled water), finally the sections were stained by hematoxylin and eosin (H\&E) stain for routine histological examination under light microscope (according to manufacturer instructions). This was performed using a digital camera (MD50, Mshot, China) which was mounted on a light microscope (BX40F4, Olympus, Japan).

\section{Blood sampling for inflammatory markers}

Blood samples were collected from rats under proper anesthesia from the orbital sinus to measure inflammatory markers (IL-1 $\beta$, IL-6, IL-10 and TNF- $\alpha$ ) 6 days post-surgery using ELISA technique. The samples were collected using serum separator tubes (SST) and left to coagulate at room temperature for $30 \mathrm{~min}$. Samples were centrifuged for $10 \mathrm{~min}$ at $1300 \mathrm{rpm}$. The samples were aliquoted and frozen at $-80{ }^{\circ} \mathrm{C}$ freezer for storage.

\section{Statistical analysis}

All data showed normal distribution. Descriptive statistics was used to display all the examined variables. The results were analyzed via one-way analysis of variance (ANOVA) followed by Tukey post hoc test to compare between different groups at the same time interval. All values are presented as means \pm SD. Values at $\mathrm{p}<0.05$ were considered to be statistically significant. Statistical analysis was performed using SPSS software version 23 for windows (SPSS Inc., Chicago, IL, USA). Charts were performed using GraphPad Prism version 7.00 (GraphPad Software, San Diego, CA).

\section{Result}

\section{Hematoxylin and eosin (H\&E) staining results}

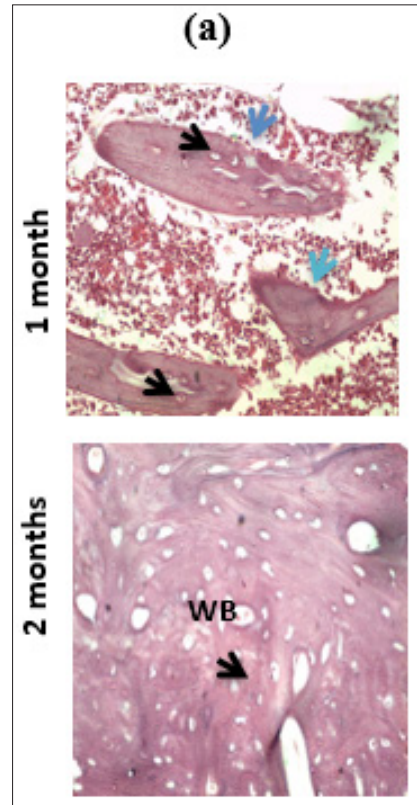

(e) (b)
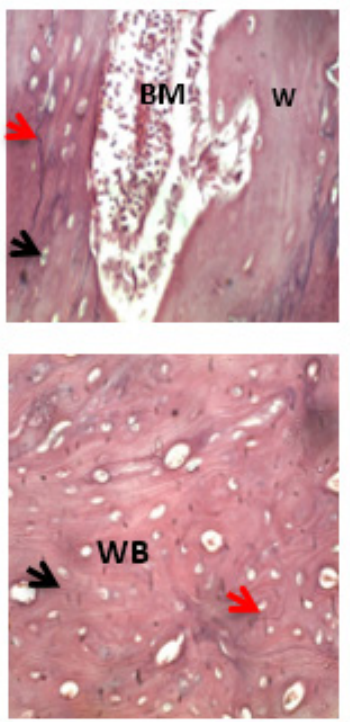

(f) (c)
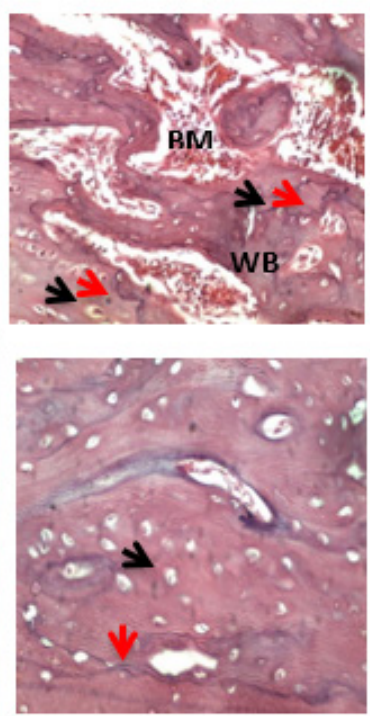

(g) (d)
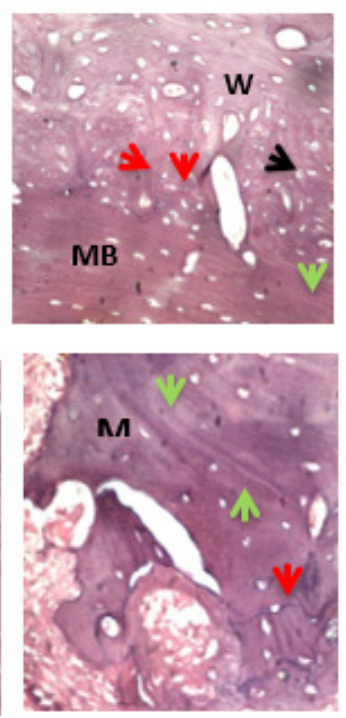

(h)

Figure 3: Histological analysis of bone specimens of the control, PRF, SIM, SIM/PRF groups one and twomonths postoperatively. (H\&E staining, original magnification $\mathrm{x} 400$ ) showing osteocytes in their dilated lacunae (black arrows), osteoblasts (blue arrows), reversal lines (red arrows), and resting lines (green arrows). BM: Bone Marrow, WB: Woven Bone, MB: Matured Bone. 
Histological evaluation of bone formation one-month postoperatively: Examination of the H\&E stained sections in bone defect area of the control group, showed limited islands of woven bone, with faintly stained matrix and dilated osteocytes lacunae. Few osteoblastic cells were inspected on the woven bone borders. The bone islands were surrounded with granulation tissue and inflammatory cells (Figure 3a).

Tissue sections obtained from the PRF group rats showed areas of woven bone with dilated osteocytes lacunae surrounding bone marrow (BM) spaces. Reversal line was hardly noticed in some specimens. Few dilated blood vessels were found in the BM spaces (Figure 3b).

Histological sections of SIM group showed apparently thick bone trabeculae compared to the PRF group. Areas of newly formed woven bone with dilated osteocytes lacunae and faintly stained matrix were found surrounding the bone marrow spaces and were intermingling with limited areas of density stained matured bone. Reversal lines were well noticed in the specimen with few resting lines (denoting bone remodeling). Vascular BM spaces were noticed with osteoblasts marinated at the borders (Figure 3c). The SIM/ PRF group histological sections showed areas of faintly stained woven bone with dilated osteocyte lacunae that were neighbored with areas of density stained matured bone with undulated resting lines. The reversal line and resting lines were observed in some areas surrounding the vascular spaces, denoting bone maturation activity (Figure 3d).

Histological evaluation of bone formation two-months postoperatively: Through examining the specimens of the control group, most of the bone defect area were filled with immature woven bone demonstrated faintly stained matrix and well observed dilated osteocyte lacunae. Hardly reversal and resting lines were observed within the specimens denoting minimal bone maturation (Figure 3e).

Concerning the PRF group, examined specimens showed areas of faintly stained woven bone were neighbored with limited areas of matured bone denoted with more darkly stained bone matrix and few resting lines surrounding vascular spaces. Few reversal lines were observed within the specimens (Figure 3f). Bone specimens of the SIM group-as compared to the previous group showed well observed darkly stained areas of matured bone neighboring to the areas of the woven bone with the characteristic dilated osteocyte lacunae were seen. Reversal lines as well as resting lines were well observed in the specimens (Figure 3g). On examining specimens of SIM/PRF group, matured bone trabeculae surrounding bone marrow spaces, appeared densely stained with normal osteocytes lacunae. Resting and reversal lines were obviously detected denoting bone remodeling activity. Some osteoblasts were found lining the BM spaces (Figure $3 \mathrm{~h}$ ).

In the current study PRF and SIM decreased significantly the pro-inflammatory IL-1 $\beta$ serum levels when compared to the control group (bone defect without intervention). SIM loaded on PRF showed the highest statistically significant decrease in IL-1 $\beta$ serum concentration $(\mathrm{P}<0.001)$ as shown in Figure 4a. Interleukin-6 and tumor necrosis factor- $\alpha$ serum levels didn't differ significantly between the control and PRF group but showed statistically significant decrease in the SIM and SIM/PRF groups (Figure $4 \mathrm{~b} \& 4 \mathrm{~d}$ ). In contrast PRF, SIM and SIM/PRF significantly increased IL-10 serum levels compared to the control group with the highest significant increase in the SIM/PRF group $(\mathrm{P}<0.001)$ (Figure $4 \mathrm{c})$.

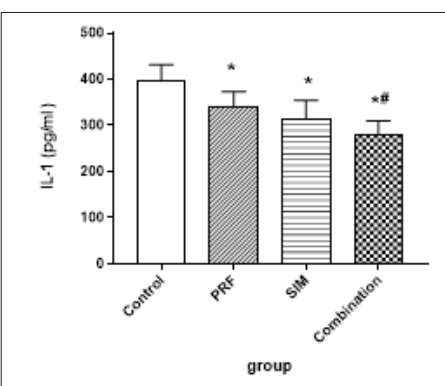

(a)

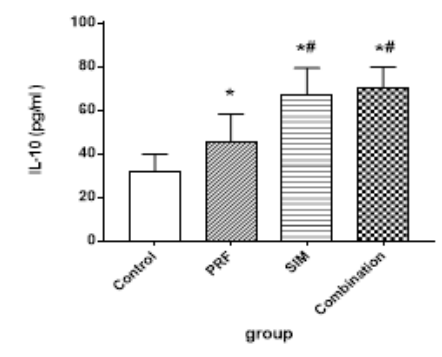

(c)

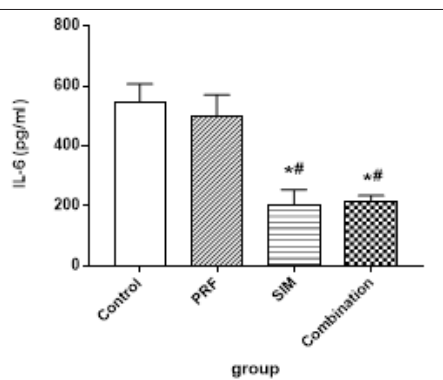

(b)

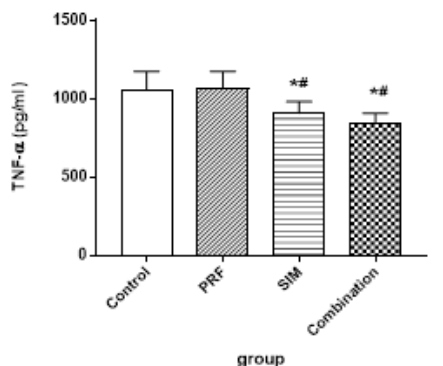

(d)

Figure 4: (a) IL-1 serum level (ng/ml), (b) IL-6 serum level (pg/ml), (c) IL-10 serum level (ng/ml), (d) TNF-a serum level (ng/ml) in the control, PRF, SIM, and SIM/PRF groups 6 days post-operatively. 


\section{Discussion}

The use of natural scaffolds as platelets rich fibrin (PRF) for autologous tissue regeneration is much favored rather than the use of synthetic foreign materials [22]. The main advantages in using the PRF are healing and bone regenerative properties in combination with its complete degradation after surgery, thus avoiding a second surgery time. Currently, it is a minimally invasive technique with low risks and satisfactory clinical results such as preventing surgical complications in patients with special health condition [23].

Although PRF has gained popularity in dental settings in recent years, only few studies in the literature have investigated its healing effects and further studies was recommended to investigate the claims made [24]. In previous study by our team [25], Platelet rich fibrin (PRF), being a biological scaffold, has proven to induce bone formation by increasing BMP-2 and VEGF at bone defect area. In addition; PRF caused significant increase in bone serum anabolic markers like OPG, OSC, and ALP. Comparing SIM with PRF each as a sole filling material in the bone defect, SIM showed more enhanced bone regeneration in addition to inhibition of bone resorption. Simvastatin (SIM) loaded on PRF has proven to cause the most significant bone formation, elevate BMP-2, VEGF proteins level and increase in bone anabolic serum markers causing earlier bone maturation than each material alone. The study suggested that SIM has more bone regeneration effect than PRF and that SIM loaded on PRF has proven to possess greater effect on induced bone defect healing and bone maturation compared to each material alone.

In the current study, the SIM group showed significant inhibitory effect on serum pro-inflammatory IL-6, IL-1 $\beta$ and TNF- $\alpha$ cytokines and stimulatory effect on the anti-inflammatory IL-10, while PRF showed only significant decrease in IL-6 and increase in IL-10 serum concentration. These results revealed that SIM has anti-inflammatory effect in addition to the enhanced bone regeneration effect. This was in accordance with [26] who supported the results of the current study and reported that SIM demonstrated significant dose-dependent inhibition of pro-inflammatory IL-6 and IL-8 production in isolated chondrocytes. The previous study concluded that SIM could play a cartilage protective role in arthritis.

Another in vitro study by [27] compared the effect of different statins on cytokine-mediated IL-6 release in SMC/MNC co-cultures. The study found that simvastatin, atorvastatin, Fluvastatin or pravastatin reduced the IL- 6 production by $53 \%, 50 \%, 64 \%$ and $60 \%$, respectively. Furthermore, the current results were in consistence with a clinical study investigated the effect of SIM and ezetimibe on IL-6 in healthy men without apparent cardiovascular disease. The study reported that SIM decreased the pro-inflammatory marker IL-6 while ezetimibe monotherapy or the combination with SIM had no effect [28].

Jameel et al. [29], investigated atorvastatin, lovastatin, and simvastatin for their modulatory effects on human peripheral blood mononuclear cells (PBMC) viability, cytokine profiles, and T-cell proliferation. The study results showed that SIM significantly decreased IL- $1 \beta$ expression to levels similar to those induced by conventional immunosuppressive drugs as dexamethasone and cyclosporine. Atorvastatin and lovastatin also decreased IL-1 $\beta$ expression, although to a lesser degree.

Clinical studies reported that PRF, clinically, can decrease pain and swelling after surgery upon local application. Post-surgical pain was mainly measured by means of need for consuming non-steroidal anti-inflammatory drugs (NSAIDs) and clinical inflammation was evaluated in terms of presence and extension of intra and extra-oral edema which was reduced with the use of PRF [30]. Ozgul et al. [20] further explained that the enhanced soft and hard tissue healing observed in their study may be attributed to the intrinsic properties of the PRF fibrin matrix, cellular-and growth factor-rich content, as well as the use of PRF as a covering membrane highlighting that it is a well-established basic surgical principle for healing is adequate blood supply and protection of the surgical site. No clear studies till now, however, indicated that it decreases serum inflammatory markers [20].

The combination group, in the present study, didn't show significant effect on the pro-inflammatory or the anti-inflammatory interleukins. This is may be attributed to the non-significant effect of PRF on some of the serum inflammatory markers. In the light of the results of the present study platelet rich fibrin (PRF), being a biological scaffold, has proven to induce bone formation.

\section{References}

1. Zhou Q, Liao JK (2009) Statins and cardiovascular diseases: from cholesterol lowering to pleiotropy. Curr Pharm Des 15(5): 467-478.

2. Di Benedetto A, Gigante I, Colucci S, Grano M (2013) Periodontal disease: Linking the primary inflammation to bone loss. Clin Dev Immunol 2013: 503754 .

3. Hikita A, Yana I, Wakeyama H, Nakamura M, Kadono Y, et al. (2006) Negative regulation of osteoclastogenesis by ectodomain shedding of receptor activator of NF-kappaB ligand. J Biol Chem 281(48): 3684636855.

4. Hofbauer LC, Schoppet M (2018) Clinical implications of the osteoprotegerin/RANKL/RANK system for bone and vascular diseases. JAMA 292(4): 490-495.

5. De Araújo RF, Souza TO, De Moura LM, Torres KP, De Souza LB, et al. (2013) Atorvastatin decreases bone loss, inflammation and oxidative stress in experimental periodontitis. PLoS One 8(10): e75322.

6. Chang B, Yang J, Li H, Lu S, Chen L, et al. (2011) Effects of atorvastatin on bone metabolism and bone mineral density in Wistar rats. Pharmazie 66(7): 535-537.

7. Deng P, Zhao S, Dai H, Guan X, Huang H (2006) Atorvastatin reduces the expression of COX-2 mRNA in peripheral blood monocytes from patients with acute myocardial infarction and modulates the early inflammatory response. Clin Chem 52(2): 300-303.

8. Hernández C, Francisco G, Ciudin A, Chacón P, Montoro B, et al. Effect of atorvastatin on lipoprotein (a) and interleukin-10: A randomized placebo-controlled trial. Diabetes Metab 37(2): 124-130.

9. Cui JJ, Wang D, Gao F, Li YR (2012) Effects of atorvastatin on pathological changes in brain tissue and plasma MMP-9 in rats with intracerebral hemorrhage. Cell Biochem Biophys 62(1): 87-90.

10. Kim J, Lee EY, Lee EB, Lee YJ, Yoo H, et al. (2012) Atorvastatin inhibits osteoclastogenesis by decreasing the expression of RANKL in the synoviocytes of rheumatoid arthritis. Arthritis Res Ther 14(4): R187. 
11. Choukroun J, Diss A, Simonpieri A, Girard MO, Schoeffler C, et al. (2006) Platelet-rich fibrin (PRF): A second-generation platelet concentrate. Part V: histologic evaluations of PRF effects on bone allograft maturation in sinus lift. Oral Surg Oral Med Oral Pathol Oral Radiol Endod 101(3): 299-303.

12. Giannini S, Cielo A, Bonanome L, Rastelli C, Derla C, et al. (2015) Comparison between PRP, PRGF and PRF: Lights and shadows in three similar but different protocols. Eur Rev Med Pharmacol Sci 19(6): 927 930.

13. Guo S, Dipietro LA (2010) Factors affecting wound healing. J Dent Res 89(3): 219-229.

14. Orsini G, Pagella P, Putignano A, Mitsiadis TA (2018) Novel biological and technological platforms for dental clinical use. Front Physiol 9: 1102.

15. Elgali I, Omar O, Dahlin C, Thomsen P (2017) Guided bone regeneration: Materials and biological mechanisms revisited. Eur J Oral Sci 125(5): 315-337.

16. Chen F-M, Liu X (2016) Advancing biomaterials of human origin for tissue engineering. Prog Polym Sci 53: 86-168.

17. Masuki H, Okudera T, Watanebe T, Suzuki M, Nishiyama K, et al. (2016) Growth factor and pro-inflammatory cytokine contents in platelet-rich plasma (PRP), plasma rich in growth factors (PRGF), advanced plateletrich fibrin (A-PRF), and concentrated growth factors (CGF). Int J Implant Dent 2(1): 19.

18. Piccin A, Di Pierro AM, Canzian L, Primerano M, Corvetta D, et al. (2017) Platelet gel: A new therapeutic tool with great potential. Blood Transfus 15(4): 333-340.

19. Borie E, Oliví DG, Orsi IA, Garlet K, Weber B, et al. (2015) Platelet-rich fibrin application in dentistry: A literature review. Int J Clin Exp Med 8(5): 7922-7929.

20. Ozgul O, Senses F, Er N, Tekin U, Tuz HH, et al. (2015) Efficacy of platelet rich fibrin in the reduction of the pain and swelling after impacted third molar surgery: Randomized multicenter split-mouth clinical trial. Head Face Med 11: 37.
21. Bancroft JD, Suvarna SK, Layton C (2012) Bancroft's theory and practice of histological techniques $\left(7^{\text {th }}\right.$ edn), Elsevier, Pheladelphia, Churchill livingstone, Pennsylvania, USA.

22. Prakash S, Thakur A (2011) Platelet concentrates: Past, present and future. J Maxillofac Oral Surg 10(1): 45-49.

23. Cortese A, Pantaleo G, Borri A, Caggiano M, Amato M (2016) Plateletrich fibrin (PRF) in implant dentistry in combination with new bone regenerative technique in elderly patients. Int J Surg Case Rep 28: 52-56.

24. Baslarli O, Tumer C, Ugur O, Vatankulu B (2015) Evaluation of osteoblastic activity in extraction sockets treated with platelet-rich fibrin. Med Oral Patol Oral Cir Bucal 20(1): e111-116.

25. Raafat SN, Amin RM, Elmazar MM, Khattab MM, El-Khatib AS (2018) The sole and combined effect of simvastatin and platelet rich fibrin as a filling material in induced bone defect in tibia of albino rats. Bone 117: 60-69.

26. Dombrecht EJ, Van Offel J, Bridts C, Ebo D, Seynhaeve V, et al. (2007) Influence of simvastatin on the production of pro-inflammatory cytokines and nitric oxide by activated human chondrocytes. Clin Exp Rheumatol 25(4): 534-539.

27. Loppnow H, Zhang L, Buerke M, Lautenschlager M, Chen L, et al. (2011) Statins potently reduce the cytokine-mediated IL- 6 release in SMC/MNC cocultures. J Cell Mol Med 15(4): 994-1004.

28. Berthold HK, Berneis K, Mantzoros CS, Krone W, Gouni BI (2013) Effects of simvastatin and ezetimibe on interleukin- 6 and high-sensitivity C-reactive protein. Scand Cardiovasc J Suppl 47(1): 20-27.

29. Jameel A, Ooi KG J, Jeffs NR, Galatowicz G, Lightman SL, et al. (2013) Statin modulation of human T-Cell proliferation, IL-1 $\beta$ and IL-17 production, and IFN- $\gamma$ T cell expression: Synergy with conventional immunosuppressive agents. Int J Inflam 2013: 434586.

30. Choukroun J, Diss A, Simonpieri A, Girard MO, Schoeffler C, et al. (2006) Platelet-rich fibrin (PRF): A second-generation platelet concentrate. Part IV: Clinical effects on tissue healing. Oral Surg Oral Med Oral Pathol Oral Radiol Endod 101(3): e56-e60. 